\title{
El paradigma económico de la educación desde la teoría de Eric A. Hanushek
}

\section{The economic paradigm of education from the theory of Eric A. Hanashek}

\author{
Dani Oved Ochoa Cervantez \\ daniovedochoacervantez@yahoo.com \\ Emilio Gabriel Esbeih Castellanos \\ egesbeih@yahoo.com \\ Junior Francisco Días Ríos \\ juniorf_dias@yahoo.com
}

Resumen

Este ensayo analiza los paradigmas de Eric A. Hanushek sobre la economía de la educación. Inicialmente, aborda la relación del capital humano con el crecimiento económico sostenido de los países, es decir, cómo el Producto Interno Bruto (PIB) se encuentra fuertemente relacionado con las mejoras de las habilidades cognitivas que posee su población, asimismo, plantea los beneficios económicos potenciales que deberían motivar a los gobiernos a mejorar la calidad de las instituciones educativas y por tanto propiciar las grandes reformas. Además, establece que una estrategia eficaz para el desarrollo de los países no solo debería centrarse en las estadísticas sobre los niños que ingresan a las escuelas o la cantidad de niños que adquieren el grado inmediato superior, sino también en mejorar las habilidades cognitivas medidas a través de las pruebas internacionales de matemáticas y ciencias. Basado en evidencia, Hanushek sostiene que la calidad de la educación medida por las habilidades cognitivas que los estudiantes obtienen en las pruebas internacionales, es sustancialmente más importante que la mera cantidad de educación, por lo tanto, plantea que invertir grandes cantidades del PIB en educación no asegura el crecimiento económico; para lograrlo, se debe incidir en los factores que son determinantes para el logro de la calidad de la educación.

Recibido 22 de mayo de 2017 /Aceptado 3 de junio de 2017. la Universidad Pedagógica Nacional Francisco Morazán, de Honduras, se comparten bajo términos de la Licencia Creative Commons: Se permite que otros puedan descargar las obras y compartirlas con otras personas, siempre y
cuando se reconozca su autoría, pero no se pueden cambiar de ninguna manera ni se pueden utilizer comercialmente. 
Palabras Clave: Economía de la educación, Habilidades Cognitivas, Crecimiento Económico,
Capital Humano, Escolaridad, Calidad de la educación, Inversión en educación.

Abstract

This essay analyzes the paradigms of Eric A. Hanushek on the economics of education. Initially, it addresses the relationship between human capital and sustained economic growth in countries, that is, how the Gross Domestic Product (GDP) is strongly related to the improvements in the cognitive abilities of its population, as well as the economic benefits Potential that should motivate governments to improve the quality of educational institutions and thus foster major reforms. In addition, it states that an effective strategy for the development of countries should not only focus on statistics on children entering schools or the number of children who acquire the next higher grade, but also on improving the cognitive skills measured through Of international math and science tests. Based on evidence, Hanushek argues that the quality of education measured by students' cognitive abilities in international tests is substantially more important than the mere amount of education, therefore it is argued that investing large amounts of GDP in education does not Ensures economic growth; To achieve this, we must focus on the factors that are determinant for the achievement of the quality of education.

Keywords: Economics of Education, Cognitive Skills, Economic Growth, Human Capital, Schooling, Quality of Education, Investment in Education.

\section{Introducción}

Hoy en día es casi imposible encontrarse a un gobernante de cualquier región del mundo que desconozca y no destaque la importancia de la educación, de hecho, existe un reconocimiento explícito e incluso políticas que afirman compromisos con el mejoramiento de la calidad educativa. Estos planteamientos no siempre se convierten en acciones y los recursos públicos 
acaban por orientarse a otras áreas, no menos importantes, tales como la seguridad y la salud, quizá subestimando el valor de las habilidades que debe adquirir la población o bien porque los resultados de esta inversión tienen un impacto a largo plazo y no son lo suficientemente visibles para poder utilizarse como capital político en las elecciones.

En la agenda internacional el acceso a la educación es una de las grandes prioridades y existe un alto compromiso internacional para lograr el segundo objetivo de Desarrollo del Milenio el cual consiste, según la Organización de Naciones Unidas (2015), en lograr la educación primaria universal. No hay duda de los resultados positivos que se han obtenido hasta la fecha, no obstante, este éxito se ha enfocado únicamente en la medición de factores de cobertura y de matrícula, haciendo de lado las ventajas de las habilidades cognitivas que facilitan la adquisición de competencias profesionales importantes para el desarrollo en el plano individual y social.

A pesar de los avances, según un estudio publicado por el Banco Mundialen América Latina existen unos 20 millones de jóvenes, entre 15 y 24 años, que ni trabajan ni estudian, los cuales forman parte del denominado grupo de los ninis. El estudio plantea que la presencia de ninis tiene efectos negativos a largo plazo en la productividad, reduciendo los salarios y las oportunidades de empleo a lo largo del ciclo de vida de las personas, obstaculizando el crecimiento económico en general (De Hoyos, Rogers y Székely, 2016). A nivel de sociedad, el efecto adverso de los ninis en los ingresos no sólo reduce el producto total de la economía debido a una menor productividad laboral (el motor del crecimiento), sino que además, obstaculiza la igualdad. la Universidad Pedagógica Nacional Francisco Morazán, de Honduras, se comparten bajo términos de la Licencia Creative Commons: Se permite que otros puedan descargar las obras y compartirlas con otras personas, siempre y
cuando se reconozca su autoría, pero no se pueden cambiar de ninguna manera ni se pueden utilizer comercialmente. 


\section{Economía de la educación}

Desde el punto de vista económico, el gasto en educación de las personas ha demostrado ser una buena inversión, es decir, desde que economistas como Schultz (1961) y Becker $(1964,1993)$ sentaron las bases de la teoría del capital humano, se sabe que las inversiones en las personas, así como las inversiones en infraestructura o capital, producen retornos positivos. La inversión en educación, salud y protección social beneficia tanto al individuo en términos de sus ingresos y oportunidades de desarrollo laboral así como a la sociedad en su conjunto (Santibañez, 2009).

La teoría del Capital Humano de Becker (1964, 1993), puso de manifiesto que un individuo mejor formado recibirá más ingresos a lo largo de su ciclo de vida. Desde la consolidación de esta teoría, objetivos como la reducción del fracaso escolar, el aumento del rendimiento académico, la mejora en la calidad de la enseñanza y la igualdad de oportunidades educativas para todos los alumnos cobraron más relevancia y han constituido el foco central de las aspiraciones en educación a nivel mundial (Santín y Valiño, 2003).

En esta línea, se dice que la economía de la educación surge de la mano de la teoría del capital humano (Becker, 1993), irrumpiendo en la educación comparada aportando indicadores y métodos cuantitativos (García, 2013), además, es considerada una ciencia de la educación que aborda una serie de fenómenos educativos desde un enfoque meramente económico y se ocupa del estudio de la realidad educativa mediante el uso de la teoría económica. Bajo estos planteamientos, la economía de la educación es concebida como la aplicación de los principios económicos, conceptos y leyes al proceso de educación y rama de la economía que utiliza las herramientas económicas para resolver problemas educativos (Pineda, 2000).

Además, la economía de la educación implica observar una diversa gama de teorías económicas, modelos y metodologías cuantitativas con la finalidad de comprender, analizar y mejorar el desempeño de los sistemas educativos. Siguiendo el concepto de economía que expone Samuelson (1955), la economía de la educación consiste en analizar como los administradores del sector educativo gestionan eficientemente los escasos recursos que existen en los sistemas educativos.

<Paradigma> - Revista de Investigación Educativa. Año 24. No. 37 


\section{Las habilidades cognitivas}

Una serie de estudios han demostrado la importancia que tienen las habilidades cognitivas que poseen los habitantes de un país, sobre todo cuando se les correlaciona con otras variables de carácter micro y macroeconómicas (Hanushek y Kimko, 2000; Hanushek y Woessmann, 2008), aquellos países que fomentan altos niveles de habilidades cognitivas en su población son los que alcanzan el nivel más alto de prosperidad a largo plazo.

Estas investigaciones sugieren que los Estados deben garantizar que todos sus ciudadanos cuenten con las competencias (habilidades cognitivas) necesarias para competir en una sociedad moderna e integrada, además, las implicaciones de la educación van más allá del impacto sobre la capacidad individual de competir, la investigación macroeconómica actual sobre el crecimiento de las naciones indica que una economía futura estable depende de las habilidades cognitivas de sus trabajadores (Hanushek y Woessmann, 2012), asimismo, las habilidades que poseen los trabajadores, aunque no son el único insumo, son un factor cada vez más importante en el crecimiento económico de un país, generando un aumento en las ganancias de los individuos y seguidamente en la economía nacional.

Hace unos años era casi imposible medir las habilidades que poseen los habitantes de un país, lo cual dificultaba confirmar su relación con otras variables, hoy en día existen pruebas internacionales en diversas áreas que ofrecen una medida confiable sobre las habilidades cognitivas que posee la población (Hanushek y Woessmann, 2012).Estas pruebas son lideradas desde el Programa para la Evaluación Internacional de Alumnos de la Organización para la Cooperación y el Desarrollo Económicos (OCDE) y son conocidas por sus siglas en inglés como PISA, persiguen evaluar los "conocimientos y habilidades necesarios para la participación plena en la sociedad del saber. PISA saca a relucir aquellos países que han alcanzado un buen rendimiento y, al mismo tiempo, un reparto equitativo de oportunidades de aprendizaje..." (OCDE, 2017). Estas pruebas son aplicadas cada tres años.

\section{Las habilidades cognitivas y el crecimiento económico}

Para Hanusheky Woessmann(2007a) los habitantes de América Latina tienen un grado de educación relativa, pero él cuestiona la razón la Universidad Pedagógica Nacional Francisco Morazán, de Honduras, se comparten bajo términos de la Licencia Creative Commons: Se permite que otros puedan descargar las obras y compartirlas con otras personas, siempre y
cuando se reconozca su autoría, pero no se pueden cambiar de ninguna manera ni se pueden utilizer comercialmente. 
por la cual el crecimiento económico de estos países ha quedado rezagado en las últimas cuatro décadas, algunos más que otros, a su criterio la respuesta se puede encontrar en el desempeño de sus sistemas de educación, lo que los estudiantes saben es comparativamente muy pobre. A pesar que los países latinoamericanos no han participado frecuentemente en pruebas de logros estudiantiles a nivel mundial, las veces que lo han hecho, sus estudiantes siempre se ubican cerca de la parte inferior de las comparaciones mundiales (Hanushek y Woessmann, 2009).

Estos resultados son evidentes considerando el bajo nivel de habilidades cognitivas y el pobre desempeño de crecimiento económico de América Latina en comparación con los resultados de otras regiones del mundo en las últimas cuatro décadas. De igual forma, los resultados son producto del mal desempeño en términos de calidad educativa de la región, descuidando las habilidades cognitivas, ya que estas son relevantes para el crecimiento económico sólo en la medida en que se mejoran.

Los planteamientos de Hanushek sobre América Latina dejan en evidencia los resultados de diferentes estudios que refuerzan la idea de una relación fuerte entre las habilidades y el crecimiento económico, sobre los beneficios positivos que tiene un país cuando se invierte en el fortalecimiento de las habilidades cognitivas, pero son observados a largo plazo (Hanushek y Woesmann, 2007c), así mismo, y no menos importante, la relación general entre la calidad de la fuerza de trabajo de los habitantes de américa latina, medida por las pruebas de habilidad cognitiva y el crecimiento económico de la zona (Hanushek, 2003).

Estos análisis empíricos del crecimiento han proporcionado, a países como Estados Unidos, indicadores de impacto a largo plazo sobre las tasas de crecimiento de una fuerza de trabajo con habilidades variadas, medida por las puntuaciones de matemáticas y ciencias, así por ejemplo, en América Latina mejorar las habilidades cognitivas de la región tendría un impacto positivo en las tasas de crecimiento económico y sería un factor determinante para mejorar la calidad de vida de sus habitantes, además se observarían mayores tasas de invención; en las empresas se observaría una mayor capacidad de introducir nuevos métodos de producción y más sofisticados; y facilitar la introducción rápida de nuevas tecnologías (Hanushek, 2003).

<Paradigma> - Revista de Investigación Educativa. Año 24. No. 37 
Por otro lado, pasar de un nivel de calidad a otro de la fuerza de trabajo depende de la proporción de trabajadores con diferentes habilidades. Por lo tanto, el impacto de las habilidades en el PIB en cualquier momento será proporcional a los niveles medios de destreza de los trabajadores en la economía (Hanushek y Woessmann, 2012).

Este escenario, plantea que una mano de obra más calificada conduce a un alto crecimiento económico a largo plazo a través de la generación de nuevas ideas y procesos de producción, es decir, los niveles más altos de capital humano en cada país le permiten innovar, mejorar su producción e importar nuevas tecnologías.

Asimismo, en los tiempos actuales donde las naciones están plagadas de alto desempleo; si los trabajadores tienen más habilidades relacionadas con el trabajo, estarán mejor capacitados para encontrar empleo (Hanushek y Woessmann, 2012). Estos autores sugieren un enfoque en la calidad, más que en la cantidad en educación, asegurando que los estudiantes realmente aprendan. Para lograrlo se debe comprender; que los programas para mejorar las habilidades cognitivas a través de las escuelas toman tiempo para implementar y tener su impacto en los estudiantes, que el impacto económico de mejorar las habilidades no se materializa hasta que los estudiantes con mayores habilidades pasen a la fuerza de trabajo y finalmente, la economía responderá positivamente a medida que se desarrollen y apliquen nuevas tecnologías a través del aprovechamiento de las nuevas cualificaciones (Hanushek y Woessmann, 2012).

\section{Capital Humano y crecimiento económico}

Otro de los conceptos que expone Hanushek, el cual no está alejado de sus planteamiento sobre las habilidades cognitivas, es el relacionado con el capital humano, sostiene que hay estudios que demuestran que el crecimiento económico de largo plazo dependen de la calidad del capital humano de una sociedad y es un componente necesario para impulsar la innovación y adaptar nuevas tecnologías (Hanushek y Woessmann, 2008).

Para Hanusheky Woessmann (2007) la educación puede aumentar el capital humano para un mejor aprovechamiento de la fuerza de trabajo, aumentando la productividad lo que conduce a un nivel de producción elevado y sostenible, además puede aumentar la capacidad la Universidad Pedagógica Nacional Francisco Morazán, de Honduras, se comparten bajo términos de la Licencia Creative Commons: Se permite que otros puedan descargar las obras y compartirlas con otras personas, siempre y cuando se reconozca su autoría, pero no se pueden cambiar de ninguna manera ni se pueden utilizer comercialmente. 
innovadora de la mano de obra y generar conocimiento para la implementación de nuevas tecnologías, creando un escenario inmejorable para el logro del crecimiento económico.

Las afirmaciones anteriores son respaldadas por economista como Schultz (1961) y Becker $(1964,1993)$ quienes han dedicado considerable atención a la comprensión del capital humano y como esté afecta a una variedad de resultados económicos. La idea central es que los individuos toman decisiones de inversión en sí mismos a través de la escolarización. Las habilidades acumuladas que son relevantes para el mercado de trabajo a partir de estas inversiones a lo largo del tiempo representan el capital humano de un individuo (Hanushek, y Wößmann, 2007a).Así mismo, el incremento y acumulación del capital humano en la sociedad, además de generar un mayor crecimiento económico, ayuda a mejorar la distribución de los ingresos futuros y genera más oportunidades para que los hogares mejoren sus ingresos y su calidad de vida (De Hoyos, Rogers y Székely, 2016).

Por el contrario, cuando no existe avances en materia de capital humano se puede obstaculizar el crecimiento económico del país y reducir los niveles de pobreza, ¿cómo ocurre?; cuando los jóvenes deciden abandonar la escuela dejan de acumular capital humano, por tanto, es seguro que a largo plazo sean los que obtengan salarios más bajos y peores perspectivas de empleo, y si además no logran encontrar trabajo remunerado después de dejar la escuela, su situación empeora. De allí, es lógico pensar que los ninis, por ejemplo, pierden varios años de experiencia laboral, y por lo tanto de ingresos, generando limitaciones para invertir en salud, educación y en otros gastos relacionados con una vida estable (De Hoyos, Rogers y Székely, 2016).

\section{La escolaridad y capital humano}

A pesar que en los países en desarrollo se cuenta con información sobre la matrícula y logro educativo; aún se presenta limitaciones para adquirir información sobre calidad. Lo que deja en evidencia que los datos disponibles sobre las habilidades cognitivas como medida de calidad de la educación muestra sus limitaciones en los países en desarrollo; este problema de acceso a este tipo de información es mayor al que se pensaba anteriormente y es un claro indicador que las políticas dirigidas a aumentar las habilidades cognitivas no han llenado las expectativas que se propusieron (Hanusheky Woessmann, 2007b). 
Los limitados datos disponibles reflejan que comúnmente las investigaciones se han enfocado en el logro escolar, es decir, años de escolaridad, afortunadamente, en los trabajos recientes cada vez más se hace hincapié en lo que la gente sabe, sus habilidades cognitivas, lo que contrasta conel tiem po en que han pasado en las escuelas (Hanushek y Woessmann, 2012).Para la mayoría de las personas un año de escolaridad no produce las mismas habilidades cognitivas en todas partes (Hanushek, y Wößmann, 2007c).

De manera análoga, para los países en desarrollo, los retornos del crecimiento de los años de escolaridad incrementan en función de la calidad de la educación. De esta manera que se justifica que en la medida existe un sistema escolar de alta calidad, se puede mantener a los niños en la escuela durante más tiempo, pero no se justifica si el sistema escolar no desarrolla habilidades (Hanushek, y Wößmann, 2007).Por otra parte la mayoría de las estrategias de desarrollo no han garantizado mejores condiciones económicas a pesar de la expansión del logro escolar; esto se debe a la falta de atención en cuanto a calidad de la educación, sin asegurarse que los estudiantes realmente aprendan. Así mismo, existe una fuerte evidencia de que las habilidades cognitivas de la población, en lugar de la mera inscripción escolar, están fuertemente relacionadas con las ganancias individuales, la distribución del ingreso y el crecimiento económico (Hanusheky Woessmann, 2007c).

\section{Ganancia individual a largo plazo}

Como ya se ha dicho, las habilidades cognitivas tienen un claro impacto tanto en los ingresos individuales como en el crecimiento de la economía en general. Es decir; lo que los estudiantes aprenden en la escuela se refleja en futuras ganancias, su rendimiento en fuerza de trabajo $y$, en última instancia, la tasa de crecimiento del país. De igual modo los economistas han demostrado que el logro de un estudiante en la escuela afecta directamente sus ganancias durante su vida, por supuesto, sin olvidar las diferencias en la cantidad de educación, experiencia en la fuerza de trabajo y una variedad de factores que influyen también en los ingresos (Hanushek, 2003). Es decir, una mayor calidad, medida por pruebas similares a las que se están utilizando actualmente en los sistemas de rendición de cuentas en todo el mundo, está estrechamente relacionada con la productividad y los ingresos individuales (Hanusheky Woessmann, 2007b). la Universidad Pedagógica Nacional Francisco Morazán, de Honduras, se comparten bajo términos de la Licencia Creative Commons: Se permite que otros puedan descargar las obras y compartirlas con otras personas, siempre y
cuando se reconozca su autoría, pero no se pueden cambiar de ninguna manera ni se pueden utilizer comercialmente. 
Bajo este orden de cosas, los beneficios individuales que tienen las habilidades cognitivas en los ingresos individuales tienen un efecto directo positivo en la economía de cada país, esta idea es reforzada por Nickell (2004), quien considera que las diferencias en la distribución de ingresos entre países es producto de las de habilidades cognitivas que posee su población, así mismo, Hanushek y Zhang (2009) mencionan que gran parte de la variación en la dispersión de los ingresos se explica por la dispersión de las habilidades. Este debate se ha centrado especialmente en la importancia de las diferentes destrezas medidas a través de pruebas cognitivas. A medida que estos resultados están disponibles, las investigaciones han destacado el valor de las habilidades para determinar los resultados económicos de las personas.

No obstante, la mayor parte de la atención al valor de la escolaridad se ha centrado en los retornos económicos que los diferentes niveles de educación tienen para las personas, asociándolos, erróneamente, que a mayor nivel de escolaridad mayores ingresos percibirán (Hanushek, y Wößmann, 2007b), entre tanto y a criterio de autor, la escolaridad parece relevante para el crecimiento económico sólo en la medida que realmente eleva el conocimiento que los estudiantes obtienen como se muestra en las pruebas internacionales que miden las habilidades cognitivas.

\section{Calidad de la educación}

Desde la visión de Hanushek, la calidad de la educación se traduce en las habilidades cognitivas que adquieren los estudiantes, específicamente en áreas como las matemáticas y la ciencias, para mejorarlas, la investigación existente sugiere que el camino más claro es el fortalecimiento de las escuelas (Hanushek, 2003; Hanushek, 2005).Los retos en materia educativa que enfrentan la mayoría de los países a comienzos del siglo XXI, incluidos los países en desarrollo, son los relacionados con la calidad, más que con la cantidad. Hablar de calidad implica centrarse en los avances que muestran los estudiantes a medida que avanzan en la escuela, tanto en conocimiento como en el fortalecimiento de las habilidades cognitivas (Hanushek, 2003).

De igual forma, Hanushek plantea que la calidad de la educación es medible a través de pruebas internacionales que son coordinadas por agencias internacionales que miden específicamente el desempeño estudiantil en habilidades cognitivas como matemáticas y ciencias. No obstante, estas pruebas son objeto de críticas por un sector de la

<Paradigma> - Revista de Investigación Educativa. Año 24. No. 37 
comunidad académica relacionados con las políticas en educación, a criterio de éstos, las pruebas pasan por alto una serie de atributos que son importantes en el proceso de enseñanza-aprendizaje, sin embargo, a criterio de Hanushek, los resultados de las pruebas demuestran el rendimiento en términos de habilidades de los estudiantes y que finalmente son las que determinan el éxito individual y social.

A pesar de estas afirmaciones, el autor sostiene que no implica que estas pruebas internacionales sean las mejores, sin embargo miden algo real, es decir, lo realmente importante para lograr impactos positivos en otras variables, como por ejemplo, en la economía (Hanushek, 2003). El desempeño verdaderamente deprimente en las pruebas mundiales, en los países de América Latina, por ejemplo, sugiere que las mismas son simplemente demasiado difíciles para un típico estudiante de la región.

\section{Calidad y/o cantidad}

Dada la importancia de las habilidades cognitivas para el desarrollo económico, es significativo documentar la situación imperante en los países en desarrollo en lo que respecta a la cantidad y la calidad de la educación (Hanushek, y Wößmann, 2007a).Gran parte de la investigación sobre el impacto económico de la educación se ha concentrado en el papel del logro escolar, es decir, en la cantidad de educación. Este enfoque es natural porque los años de escolarización son fácilmente medibles, generando que los datos sobre los años alcanzados y la matricula estén fácilmente disponibles.

La cantidad de educación escolar es una medida muy cruda del conocimiento y habilidades cognitivas de las personas, es poco probable que lo aprendido durante el sexto grado en una cabaña rural en un país en desarrollo es igual a lo que se aprende en un sexto grado americano (Hanushek y Woessmann, 2010). Sin embargo, eso es lo que los análisis empíricos suponen implícitamente cuando se centran exclusivamente en las diferencias en los años promedio de escolaridad entre países.

A partir de estos planteamientos, el autor expone que las diferencias en los logros de aprendizaje son más determinantes para explicar las diferencias en el crecimiento entre países, que las diferencias en el número promedio de años de escolaridad o en las tasas de matrícula (Hanusheky Woessmann, 2007c). Lo que realmente preocupa subyace a la idea que los teóricos de la literatura sobre la educación se aferran a los años de la Universidad Pedagógica Nacional Francisco Morazán, de Honduras, se comparten bajo términos de la Licencia Creative Commons: Se permite que otros puedan descargar las obras y compartirlas con otras personas, siempre y
cuando se reconozca su autoría, pero no se pueden cambiar de ninguna manera ni se pueden utilizer comercialmente. 
escolaridad como una medida de la calidad educación y olvidan las diferencias cualitativas en el conocimiento. Actuar y pensar bajo esta idea, deja sin valor el núcleo central de la educación; la adquisición de las habilidades cognitivas.

Utilizar el promedio de años de escolaridad como medida de educación supone implícitamente que un año de escolaridad ofrece el mismo aumento de conocimientos y habilidades independientemente del sistema educativo (Hanusheky Woessmann, 2007c). Simplemente asistir a la escuela no es suficiente si los estudiantes no están aprendiendo a un nivel alto. Es el aprendizaje y no la asistencia que debe tener la más alta prioridad (Hanushek y Woessmann, 2012).

\section{Eficiencia en la inversión en educación}

Una de las interrogantes más discutidas en materia educativa es ¿cuánto debe invertir el Estado en educación?, no es una pregunta fácil de responder, sobre todo, teniendo en cuenta que la inversión pública en educación se realiza a expensas de otras inversiones públicas. Los trabajos de Eric Hanushek defienden la tesis que más dinero no implica por sí mismo mejores resultados. Hanushek concluye, tras una extensa revisión de trabajos, que no existe una relación significativa clara que ratifique de forma significativa que más factores productivos escolares supongan un aumento en los resultados de aprendizaje de los alumnos.

Aunque no siempre están de acuerdo, la mayoría de los estudios han encontrado que las diferencias en el nivel de gasto absoluto o en los aumentos de gasto tienen poca o ninguna relación constante con el rendimiento estudiantil (Hanushek, 2003, 2006).Este autor defiende básicamente que los mayores recursos deben de ir acompañados de cambios institucionales como la competencia entre escuelas, la medición sistematizada de los resultados y los recursos y la introducción de incentivos al profesorado.

A lo largo del último medio siglo otro tema que entrado en debate es cómo el rendimiento de los estudiantes se relaciona con el financiamiento de las escuelas, estos hechos han cambiado dramáticamente las discusiones políticas sobre la financiación escolar. También ha dejado claro que las discusiones financieras no pueden separarse de las discusiones más amplias sobre políticas educativas debido a la importancia de integrar los incentivos financieros con otras políticas diseñadas para mejorar el logro educativo.

<Paradigma> - Revista de Investigación Educativa. Año 24. No. 37 
Además, es importante destacar estos temas en la discusión de las finanzas porque las reducciones de tamaño de las clases así como los aumentos en los salarios de los maestros han sido temas muy importantes en el último medio siglo producto de los enormes incrementos en el gasto real por alumno en las escuelas (Hanushek y Rivkin, 1997), además, porque los tomadores de decisiones en la educación han evitado considerar la productividad (costo beneficio) en el sector educativo, y se han centrado simplemente encontrar maneras de aumentar los resultados (calidad) sin prestar mucha atención a los costos o los recursos necesarios, así, las discusiones sobre la productividad casi nunca han entrado en los debates sobre la política educativa.

Los programas de reducción de tamaño de clase han sido muy populares a pesar de que son quizás el más caro de todos los programas de reforma escolar y aun cuando la investigación sugiere que es poco probable que se asocian generalmente con el mejoramiento del logro estudiantil (Hanushek y Rivkin, 2004).Por otro lado, el pago a los maestros también muestra poca relación consistente con el logro. Un maestro que ha tenido éxito en mejorar el rendimiento de sus estudiantes es tan probable que tenga un salario bajo como un salario alto. Según los autores (Hanushek y Woessmann, 2011a) una alternativa potencial es alterar la estructura de la financiación escolar para ofrecer incentivos de desempeño a los maestros y otros miembros del personal escolar, así por ejemplo, el desempeño de un sistema se ve afectado por los incentivos que enfrentan los actores. Es decir, si los actores en el proceso educativo son recompensados (extrínseca o intrínsecamente) por producir mayor rendimiento estudiantil, y si son castigados por no producir altos logros, es probable que el logro mejore (Hanushek y Woessmann, 2011b).

En síntesis, no es posible esperar un mayor rendimiento de los estudiantes simplemente proporcionando recursos adicionales a las escuelas. Debe procurarse que cualquier recurso orientado a proveer incentivos de las personas en las escuelas, genere un efecto directo en la calidad de la educación, la idea central subyace a uno no puede esperar mejorar el logro estudiantil y los resultados simplemente poniendo más recursos en las escuelas existentes. Por lo tanto, el enfoque tradicional de la política de financiación de las escuelas sobre los flujos de recursos es erróneo, porque entra en conflicto con una base de resultados para la toma de decisiones. la Universidad Pedagógica Nacional Francisco Morazán, de Honduras, se comparten bajo términos de la Licencia Creative Commons: Se permite que otros puedan descargar las obras y compartirlas con otras personas, siempre y
cuando se reconozca su autoría, pero no se pueden cambiar de ninguna manera ni se pueden utilizer comercialmente. 


\section{Consideraciones finales}

La investigación económica de la última década indica que el logro educativo, medido por las pruebas de logros estudiantiles internacionales, tiene un efecto fuerte y consistente en el crecimiento económico a largo plazo de las naciones. La evidencia apunta fuertemente al énfasis en el desarrollo de habilidades básicas, lo que a su vez apunta al papel clave de la educación primaria y secundaria.

Por la experiencia vivida en los países desarrollados, se debe asumir que las habilidades desempeñan el mismo papel en el futuro que en el pasado, de modo que, los resultados pasados proporciona una manera directa de proyectar el futuro. Para tener un futuro prometedor en términos de crecimientos económicos constantes es necesario que los gobernantes mejoren las habilidades de su población, para lograrlo, se debe mejorar la calidad de las escuelas y sin duda alguna establecer un conjunto de políticas públicas educativas a largo plazo y coherente con el objetivo que se persigue.

Si la educación tiene un impacto tan dramático en la productividad económica y el crecimiento de un país, ¿Cuáles son las implicaciones para los países de América Latina, que poseen un sistema educativo débil? ¿Existe la posibilidad de avanzar cuando el desempeño de sus estudiantes en pruebas internacionales de matemáticas y ciencias han sido deficientes?, ante este panorama desalentador ,los gobiernos deben tomar su rol principal en las instituciones de enseñanza como actor principal en el establecimiento de la agenda para asegurar que se obtengan mejores resultados y lograr mejorar las habilidades cognitivas de su población.

Implica entonces, que el acceso a la educación, para los países de la región latinoamericana, debería constituir una prioridad en la agenda de desarrollo, tal como lo demuestra los "Objetivos de Desarrollo del Milenio" en donde se plantea por ejemplo, una educación primaria universal, no obstante, es urgente un enfoque en la calidad más que en la cantidad de la educación, asegurando que los estudiantes realmente aprendan. Hasta ahora, mientras América Latina ha tenido un logro escolar razonable, lo que los estudiantes saben es comparativamente muy pobre, basado en que países latinoamericanos han participado infrecuentemente en pruebas de logros estudiantiles a nivel mundial, y sus estudiantes siempre se ubican por debajo de las comparaciones mundiales. 
Aunque muchos factores ayudan a determinar las habilidades cognitivas, la mayoría de los esfuerzos gubernamentales para mejorar se centran en las escuelas, el lugar donde tienen mayor influencia política. Desafortunadamente, la reforma de las políticas escolares y el mejoramiento del desempeño no son sólo una cuestión de voluntad. Si se conociera la efectividad de diferentes recursos o combinaciones de los mismos sería fácil definir una estrategia de reforma óptima. El problema es que actualmente no tenemos suficiente conocimiento creíble sobre la mejor manera de usar nuevos recursos.

Por consiguiente, una estrategia educativa eficaz para el desarrollo debería centrarse no solo en el envío de más niños a la escuela, ya que a menudo se interpreta el segundo Objetivo de Desarrollo del Milenio, sino también en el mantenimiento o la mejora de la calidad de la escolarización. La tarea que nos ocupa es imponente. Como muestra la encuesta PISA, las disparidades en la educación secundaria entre los países en desarrollo y los países de la OCDE son aún mayores cuando se considera no sólo el acceso, sino también los logros de aprendizaje, asistir a la escuela afecta los resultados económicos sólo en la medida en que realmente añade al aprendizaje de los estudiantes. El nivel de escolaridad ni siquiera tiene una relación significativa con el crecimiento económico después de que uno explique las habilidades cognitivas.

Además, reducir las disparidades en el acceso y en la calidad de la educación son dos objetivos que deben perseguirse simultáneamente para que cualquier reforma educativa tenga éxito, se han logrado progresos considerables en el aumento de la matrícula, pero podrían ser mejores si las personas que dirigen la educación se dieran cuenta que recibir una baja calidad de la educación repercute en el rendimiento económico futuro a nivel individual y de país.

Se debe reconocer que las deficiencias en los sistemas educativos es producto de muchos factores, pero los países deberían investigar cuáles son las causas precisas en su propio contexto y deberían alentarse a experimentar para encontrar la mejor manera de corregir las debilidades. Herramientas como la certificación eficaz de los maestros, la divulgación pública de los logros educativos de las escuelas y de los maestros, el control escolar local por parte de las asociaciones de padres y, más en general, todas las medidas que contribuyen a la rendición de cuentas de los maestros y directores. la Universidad Pedagógica Nacional Francisco Morazán, de Honduras, se comparten bajo términos de la Licencia Creative Commons: Se permite que otros puedan descargar las obras y compartirlas con otras personas, siempre y
cuando se reconozca su autoría, pero no se pueden cambiar de ninguna manera ni se pueden utilizer comercialmente. 
No menos importante es entender las implicaciones de las políticas diseñadas para mejorar los resultados educativos. Las evidencias aquí planteadas proporcionan información sobre los beneficios económicos a largo plazo de las mejoras en la calidad de la educación, para conseguirlo, es útil relacionar las reformas de política educativa directamente con el patrón de resultados económicos futuros que se desean lograr.

Estas aspiraciones no pueden lograrse instantáneamente, pero requiere de cambios en las escuelas, por ejemplo; mediante el reem plazo sistemático de los maestros por medio del retiro y posterior contratación. Finalmente, se debe estar consciente que si las reformas tienen éxito, su impacto en la economía no será inmediato y pasará algún tiempo para observar mejoras, las recompensas son grandes, pero las políticas deben ser consideradas a largo plazo y sin duda alguna se requiere paciencia, la cual no siempre se tiene al formular políticas educativas. 


\section{Referencias Bibliográficas}

Becker, G. (1964). Human Capital: A Theoretical and Empirical Analysis with Special Reference to Education. Columbia University Press. New York.

Becker, G. S. (1993). Human capital. Chicago: University of Chicago Press.

De Hoyos, R., Rogers, H., y Székely, M. (2016). Ninis en América latina: 20 millones de jóvenes en busca de oportunidades. Banco Mundial, Washington, DC. Licencia: Creative Commons de Reconocimiento CC BY 3.0 IGO.

García A. (2013). Aportes de la Economía a la Educación Comparada. Recuperado el 14 de Abril de 2017, de https://www.researchgate.net/ publication/272823852_Aportes_de_la_economia_a_la_educacion_comparada.

Hanushek, E, A., y Woessmann. L. (2011a). The economics of international differences in educational achievement. In E. A. Hanushek. S. Machin, \& L. Woessmann (Eds.), Handbook of the economics of education (Vol. 3, pp. 89-200). Amsterdam, Netherlands: North Holland.

Hanushek, E. A. (2003). The failure of input-based schooling policies. Economic Journal, (485), 64-98.

Hanushek, E. A. (2006). School resources. In E. A. Hanushek \& F. Welch (Eds.), Handbook of the economics of education (pp. 865-908). Amsterdam, Netherlands: North Holland.

Hanushek, E. A. y D. D. Kimko (2000), "Schooling, Labor Force Quality, and the Growth of Nations", American Economic Review 90, 1184-1208.

Hanushek, E. A. y L. Woessmann (2010). The High Cost of Low Educational Performance: The Long-Run Economic Impact of Improving PISA Outcomes, Organization for Economic Cooperation and Development, Paris. la Universidad Pedagógica Nacional Francisco Morazán, de Honduras, se comparten bajo términos de la Licencia Creative Commons: Se permite que otros puedan descargar las obras y compartirlas con otras personas, siempre y cuando se reconozca su autoría, pero no se pueden cambiar de ninguna manera ni se pueden utilizer comercialmente. 
Hanushek, E. A. y L. Woessmann (2011b), "How Much do Educational Outcomes Matter in OECD Countries?", Economic Policy 26, 427-491.

Hanushek, E. A. y L. Zhang (2009), "Quality-Consistent Estimates of International Schooling and Skill Gradients", Journal of Human Capital 3, 107-143.

Hanushek, E. A., y Rivkin. S. G. (2004). How to improve the supply of high quality teachers. In D. Ravitch (Ed.). Bmokings papers on education policy 2004 (pp. 7-25). Washington. DC: Brookings Institution Press.

Hanushek, Eric A. y Ludger Woessmann (2007a). Education Quality and Economic Growth. The World Bank, Washington, DC.

Hanushek, Eric A. y Ludger Woessmann. (2008). "The Role of Cognitive Skills in Economic Development." Journal of Economic Literature: 46 (3), 607-68. American Economic Association.

Hanushek, Eric A. y Ludger Woessmann. (2012). The Economic Benefit of Educational Reform in the European Union. CESifo Economic Studies, 58 (1), 73-109.

Hanushek, Eric A., y Dennis D. Kimko. (2000). "Schooling, Labor Force Quality, and the Growth of Nations." American Economic Review 90 (5): 1184-1208.

Hanushek, Eric A., y Ludger Woessmann. (2009). “Do better schools lead to more growth?" NBER, Cambridge, MA, WP 14633, National Bureau of Economic Research (January).

Hanushek, Eric y Ludger Wößmann (2007b). Education Quality and Economic Growth. Washington D.C.: TheWorld Bank.

Hanushek, Eric y Wößmann, (2007c). Lugger, Calidad de la Educación y Crecimiento Económico. (Washington, DC: PREAL.

Hanushek. E. A. (2005). Why Quality Matters in Education. Finance \& Development. 15-19.

<Paradigma> - Revista de Investigación Educativa. Año 24. No. 37 
Hanushek. E. A.y Rivkin. S. G. (1997). Understanding the twentieth century growth in U.S. school spending. Journal of Human Resources, 2(1).35-68.

Nickell, Stephen. 2004. "Poverty and Worklessness in Britain." Economic Journal 114: C1-25.

OECD. (2017). PISA en español. Recuperado de http://www.oecd.org/ pisa/pisaenespaol.htm

Organización de Nacional Unidas. (2015). Objetivos de Desarrollo del Milenio: Informe de 2015. Nueva York.

Pineda, P. (2000). Economía de la educación: una disciplina Pedagógica en pleno desarrollo. BIBLID, 12, 143-158.

Samuelson, Paul A. (1955). Economics, tercera edición, EUA: McGraw-Hill

Santibañez, L. (2009). El Impacto Del Gasto Sobre La Calidad Educativa. México: Programa de las Naciones Unidas para el Desarrollo.

Santín, D. y Valiño A. (2003). La Función De Producción Educativa: ¿Importan Las Escuelas? Tenerife: X Encuentro De Economía Pública.

Schultz, Theodore W. (1961) "Investment in Human Capital." The American Economic Review 51 (1), 1-17 la Universidad Pedagógica Nacional Francisco Morazán, de Honduras, se comparten bajo términos de la Licencia Creative Commons: Se permite que otros puedan descargar las obras y compartirlas con otras personas, siempre y cuando se reconozca su autoría, pero no se pueden cambiar de ninguna manera ni se pueden utilizer comercialmente. 